\title{
Probability Measure on Discrete Spaces and Algebra of Real-Valued Random Variables
}

\author{
Hiroyuki Okazaki \\ Shinshu University \\ Nagano, Japan
}

\author{
Yasunari Shidama \\ Shinshu University \\ Nagano, Japan
}

\begin{abstract}
Summary. In this article we continue formalizing probability and randomness started in [13], where we formalized some theorems concerning the probability and real-valued random variables. In this paper we formalize the variance of a random variable and prove Chebyshev's inequality. Next we formalize the product probability measure on the Cartesian product of discrete spaces. In the final part of this article we define the algebra of real-valued random variables.
\end{abstract}

MML identifier: $\underline{\text { RANDOM_2, }}$ version: $\underline{7.11 .07 \quad 4.146 .1112}$

The notation and terminology used here have been introduced in the following papers: [21], [3], [16], [1], [9], [17], [14], [4], [5], [11], [15], [6], [12], [22], [13], [19], [20], [8], [10], [18], [2], and [7].

\section{VARIANCE}

In this paper $O_{1}$ denotes a non empty set, $r$ denotes a real number, $S_{1}$ denotes a $\sigma$-field of subsets of $O_{1}$, and $P$ denotes a probability on $S_{1}$.

One can prove the following two propositions:

(1) For every one-to-one function $f$ and for all subsets $A, B$ of $\operatorname{dom} f$ such that $A$ misses $B$ holds $\operatorname{rng}(f\lceil A)$ misses $\operatorname{rng}(f\lceil B)$.

(2) For all functions $f, g$ holds $\operatorname{rng}(f \cdot g) \subseteq \operatorname{rng}(f\lceil\operatorname{rng} g)$.

Let us consider $O_{1}, S_{1}$. Observe that there exists a real-valued random variable of $S_{1}$ which is non-negative.

Let us consider $O_{1}, S_{1}$ and let $X$ be a real-valued random variable of $S_{1}$. Note that $|X|$ is non-negative.

The following propositions are true: 
(3) $O_{1} \longmapsto 1=\chi_{\left(O_{1}\right), O_{1}}$.

(4) $O_{1} \longmapsto r$ is a real-valued random variable of $S_{1}$.

(5) For every non empty set $X$ and for every partial function $f$ from $X$ to $\mathbb{R}$ holds $f^{2}=(-f)^{2}$ and $f^{2}=|f|^{2}$.

(6) Let $X$ be a non empty set and $f, g$ be partial functions from $X$ to $\mathbb{R}$. Then $(f+g)^{2}=f^{2}+2(f g)+g^{2}$ and $(f-g)^{2}=\left(f^{2}-2(f g)\right)+g^{2}$.

Let us consider $O_{1}, S_{1}, P$ and let $X$ be a real-valued random variable of $S_{1}$. Let us assume that $X$ is integrable on $P$ and $|X|^{2}$ is integrable on P2M $P$. The functor variance $(X, P)$ yielding an element of $\mathbb{R}$ is defined by the condition (Def. 1).

(Def. 1) There exists a real-valued random variable $Y$ of $S_{1}$ and there exists a real-valued random variable $E$ of $S_{1}$ such that $E=O_{1} \longmapsto E_{P}\{X\}$ and $Y=X-E$ and $Y$ is integrable on $P$ and $|Y|^{2}$ is integrable on P2M $P$ and $\operatorname{variance}(X, P)=\int|Y|^{2} \mathrm{~d} \mathrm{P} 2 \mathrm{M} P$.

\section{Chebyshev's Inequality}

One can prove the following proposition

(7) Let given $O_{1}, S_{1}, P, r$ and $X$ be a real-valued random variable of $S_{1}$. Suppose $0<r$ and $X$ is non-negative and $X$ is integrable on $P$ and $|X|^{2}$ is integrable on P2M $P$. Then $P\left(\left\{t \in O_{1}: r \leq\left|X(t)-E_{P}\{X\}\right|\right\}\right) \leq$ $\frac{\operatorname{variance}(X, P)}{r^{2}}$.

\section{Product Probability Measure}

The following propositions are true:

(8) Let $O_{1}$ be a non empty finite set, $f$ be a function from $O_{1}$ into $\mathbb{R}$, and $P$ be a function from $2^{O_{1}}$ into $\mathbb{R}$. Suppose that

(i) for every set $x$ such that $x \subseteq O_{1}$ holds $0 \leq P(x) \leq 1$,

(ii) $P\left(O_{1}\right)=1$, and

(iii) for every finite subset $z$ of $O_{1}$ holds $P(z)=\operatorname{setopfunc}\left(z, O_{1}, \mathbb{R}, f,+_{\mathbb{R}}\right)$. Then $P$ is a probability on the trivial $\sigma$-field of $O_{1}$.

(9) Let $D_{1}$ be a non empty set, $F$ be a function from $D_{1}$ into $\mathbb{R}$, and $Y$ be a finite subset of $D_{1}$. Then there exists a finite sequence $p$ of elements of $D_{1}$ such that $p$ is one-to-one and $\operatorname{rng} p=Y$ and $\operatorname{setopfunc}\left(Y, D_{1}, \mathbb{R}, F,+\mathbb{R}\right)=$ $\sum \operatorname{FuncSeq}(F, p)$.

(10) Let $D_{1}$ be a non empty set, $F$ be a function from $D_{1}$ into $\mathbb{R}, Y$ be a finite subset of $D_{1}$, and $p$ be a finite sequence of elements of $D_{1}$. If $p$ is one-to-one and $\operatorname{rng} p=Y$, then $\operatorname{setopfunc}\left(Y, D_{1}, \mathbb{R}, F,+_{\mathbb{R}}\right)=\sum \operatorname{FuncSeq}(F, p)$. 
(11) Let $D_{2}, D_{3}$ be non empty sets, $F_{1}$ be a function from $D_{2}$ into $\mathbb{R}, F_{2}$ be a function from $D_{3}$ into $\mathbb{R}, G$ be a function from $D_{2} \times D_{3}$ into $\mathbb{R}, Y_{1}$ be a non empty finite subset of $D_{2}$, and $p_{1}$ be a finite sequence of elements of $D_{2}$. Suppose $p_{1}$ is one-to-one and $\operatorname{rng} p_{1}=Y_{1}$. Let $p_{2}$ be a finite sequence of elements of $D_{3}, p_{3}$ be a finite sequence of elements of $D_{2} \times D_{3}, Y_{2}$ be a non empty finite subset of $D_{3}$, and $Y_{3}$ be a finite subset of $D_{2} \times D_{3}$. Suppose that

(i) $p_{2}$ is one-to-one,

(ii) $\operatorname{rng} p_{2}=Y_{2}$,

(iii) $p_{3}$ is one-to-one,

(iv) $\operatorname{rng} p_{3}=Y_{3}$,

(v) $Y_{3}=Y_{1} \times Y_{2}$, and

(vi) for all sets $x, y$ such that $x \in Y_{1}$ and $y \in Y_{2}$ holds $G(x, y)=F_{1}(x)$. $F_{2}(y)$.

Then $\sum \operatorname{FuncSeq}\left(G, p_{3}\right)=\sum \operatorname{FuncSeq}\left(F_{1}, p_{1}\right) \cdot \sum \operatorname{FuncSeq}\left(F_{2}, p_{2}\right)$.

(12) Let $D_{2}, D_{3}$ be non empty sets, $F_{1}$ be a function from $D_{2}$ into $\mathbb{R}, F_{2}$ be a function from $D_{3}$ into $\mathbb{R}, G$ be a function from $D_{2} \times D_{3}$ into $\mathbb{R}, Y_{1}$ be a non empty finite subset of $D_{2}, Y_{2}$ be a non empty finite subset of $D_{3}$, and $Y_{3}$ be a finite subset of $D_{2} \times D_{3}$. Suppose $Y_{3}=Y_{1} \times Y_{2}$ and for all sets $x, y$ such that $x \in Y_{1}$ and $y \in Y_{2}$ holds $G(x, y)=F_{1}(x) \cdot F_{2}(y)$. Then setopfunc $\left(Y_{3}, D_{2} \times D_{3}, \mathbb{R}, G,+_{\mathbb{R}}\right)=\operatorname{setopfunc}\left(Y_{1}, D_{2}, \mathbb{R}, F_{1},+_{\mathbb{R}}\right)$. $\operatorname{setopfunc}\left(Y_{2}, D_{3}, \mathbb{R}, F_{2},+_{\mathbb{R}}\right)$.

(13) Let $D_{1}$ be a non empty set, $F$ be a function from $D_{1}$ into $\mathbb{R}$, and $Y$ be a finite subset of $D_{1}$. If for every set $x$ such that $x \in Y$ holds $0 \leq F(x)$, then $0 \leq \operatorname{setopfunc}\left(Y, D_{1}, \mathbb{R}, F,+_{\mathbb{R}}\right)$.

(14) Let $D_{1}$ be a non empty set, $F$ be a function from $D_{1}$ into $\mathbb{R}$, and $Y_{1}, Y_{2}$ be finite subsets of $D_{1}$. Suppose $Y_{1} \subseteq Y_{2}$ and for every set $x$ such that $x \in Y_{2}$ holds $0 \leq F(x)$. Then setopfunc $\left(Y_{1}, D_{1}, \mathbb{R}, F,+_{\mathbb{R}}\right) \leq$ $\operatorname{setopfunc}\left(Y_{2}, D_{1}, \mathbb{R}, F,+\mathbb{R}\right)$.

(15) Let $O_{1}$ be a non empty finite set, $P$ be a probability on the trivial $\sigma$-field of $O_{1}, Y$ be a non empty finite subset of $O_{1}$, and $f$ be a function from $O_{1}$ into $\mathbb{R}$. Then there exists a finite sequence $G$ of elements of $\mathbb{R}$ and there exists a finite sequence $s$ of elements of $Y$ such that

(i) $\operatorname{len} G=\overline{\bar{Y}}$,

(ii) $s$ is one-to-one,

(iii) $\operatorname{rng} s=Y$,

(iv) $\quad$ len $s=\overline{\bar{Y}}$,

(v) for every natural number $n$ such that $n \in \operatorname{dom} G$ holds $G(n)=f(s(n))$. $P(\{s(n)\})$, and

(vi) $\int f \uparrow Y \mathrm{~d} \mathrm{P} 2 \mathrm{M} P=\sum G$.

Let $O_{2}, O_{3}$ be non empty finite sets, let $P_{1}$ be a probability on the trivial 
$\sigma$-field of $\mathrm{O}_{2}$, and let $\mathrm{P}_{2}$ be a probability on the trivial $\sigma$-field of $\mathrm{O}_{3}$. The functor Product-Probability $\left(\mathrm{O}_{2}, \mathrm{O}_{3}, \mathrm{P}_{1}, \mathrm{P}_{2}\right)$ yielding a probability on the trivial $\sigma$-field of $\mathrm{O}_{2} \times \mathrm{O}_{3}$ is defined by the condition (Def. 2).

(Def. 2) There exists a function $Q$ from $O_{2} \times O_{3}$ into $\mathbb{R}$ such that

(i) for all sets $x, y$ such that $x \in O_{2}$ and $y \in O_{3}$ holds $Q(x, y)=P_{1}(\{x\})$. $P_{2}(\{y\})$, and

(ii) for every finite subset $z$ of $O_{2} \times O_{3}$ holds (Product-Probability $\left(O_{2}, O_{3}, P_{1}\right.$, $\left.\left.P_{2}\right)\right)(z)=\operatorname{setopfunc}\left(z, O_{2} \times O_{3}, \mathbb{R}, Q,+\mathbb{R}\right)$.

Next we state two propositions:

(16) Let $O_{2}, O_{3}$ be non empty finite sets, $P_{1}$ be a probability on the trivial $\sigma$-field of $O_{2}, P_{2}$ be a probability on the trivial $\sigma$-field of $O_{3}, Y_{1}$ be a non empty finite subset of $\mathrm{O}_{2}$, and $Y_{2}$ be a non empty finite subset of $\mathrm{O}_{3}$. Then (Product-Probability $\left.\left(O_{2}, O_{3}, P_{1}, P_{2}\right)\right)\left(Y_{1} \times Y_{2}\right)=P_{1}\left(Y_{1}\right) \cdot P_{2}\left(Y_{2}\right)$.

(17) Let $\mathrm{O}_{2}, \mathrm{O}_{3}$ be non empty finite sets, $P_{1}$ be a probability on the trivial $\sigma$-field of $\mathrm{O}_{2}, P_{2}$ be a probability on the trivial $\sigma$ field of $O_{3}$, and $y_{1}, y_{2}$ be sets. If $y_{1} \in O_{2}$ and $y_{2} \in O_{3}$, then (Product-Probability $\left.\left(O_{2}, O_{3}, P_{1}, P_{2}\right)\right)\left(\left\{\left\langle y_{1}, y_{2}\right\rangle\right\}\right)=P_{1}\left(\left\{y_{1}\right\}\right) \cdot P_{2}\left(\left\{y_{2}\right\}\right)$.

\section{Algebra of Real-valued Random Variables}

Let $O_{1}$ be a non empty set and let $S_{1}$ be a $\sigma$-field of subsets of $O_{1}$. The $\mathbb{R}$-valued random variables set of $S_{1}$ yields a non empty subset of RAlgebra $O_{1}$ and is defined as follows:

(Def. 3) The $\mathbb{R}$-valued random variables set of $S_{1}=\{f: f$ ranges over real-valued random variables of $\left.S_{1}\right\}$.

Let us consider $O_{1}, S_{1}$. Note that the $\mathbb{R}$-valued random variables set of $S_{1}$ is additively-linearly-closed and multiplicatively-closed.

Let us consider $O_{1}, S_{1}$. The $\mathbb{R}$ algebra of real-valued-random-variables of $S_{1}$ yielding an algebra is defined by the condition (Def. 4).

(Def. 4) The $\mathbb{R}$ algebra of real-valued-random-variables of $S_{1}=\langle$ the $\mathbb{R}$ valued random variables set of $S_{1}$, mult(the $\mathbb{R}$-valued random variables set of $S_{1}$, RAlgebra $\left.O_{1}\right)$, Add (the $\mathbb{R}$-valued random variables set of $S_{1}$, RAlgebra $O_{1}$ ), Mult(the $\mathbb{R}$-valued random variables set of $S_{1}$, RAlgebra $O_{1}$ ), One(the $\mathbb{R}$-valued random variables set of $S_{1}$, RAlgebra $O_{1}$ ), Zero(the $\mathbb{R}$-valued random variables set of $S_{1}$, RAlgebra $\left.\left.O_{1}\right)\right\rangle$.

\section{REFERENCES}

[1] Grzegorz Bancerek and Krzysztof Hryniewiecki. Segments of natural numbers and finite sequences. Formalized Mathematics, 1(1):107-114, 1990. 
[2] Józef Białas. Series of positive real numbers. Measure theory. Formalized Mathematics, 2(1):173-183, 1991.

[3] Czesław Byliński. Binary operations. Formalized Mathematics, 1(1):175-180, 1990.

[4] Czesław Byliński. Functions and their basic properties. Formalized Mathematics, 1(1):5565, 1990.

[5] Czesław Byliński. Functions from a set to a set. Formalized Mathematics, 1(1):153-164, 1990.

[6] Czesław Byliński. Partial functions. Formalized Mathematics, 1(2):357-367, 1990.

[7] Czesław Byliński. Some basic properties of sets. Formalized Mathematics, 1(1):47-53, 1990.

[8] Czesław Byliński. The sum and product of finite sequences of real numbers. Formalized Mathematics, 1(4):661-668, 1990.

[9] Agata Darmochwał. Finite sets. Formalized Mathematics, 1(1):165-167, 1990.

[10] Jarosław Kotowicz. Real sequences and basic operations on them. Formalized Mathematics, 1(2):269-272, 1990.

[11] Keiko Narita, Noboru Endou, and Yasunari Shidama. Integral of complex-valued measurable function. Formalized Mathematics, 16(4):319-324, 2008, doi:10.2478/v10037-0080039-6.

[12] Andrzej Nędzusiak. $\sigma$-fields and probability. Formalized Mathematics, 1(2):401-407, 1990.

[13] Hiroyuki Okazaki and Yasunari Shidama. Probability on finite set and real-valued random variables. Formalized Mathematics, 17(2):129-136, 2009, doi: 10.2478/v10037-009-0014-x.

[14] Henryk Oryszczyszyn and Krzysztof Prażmowski. Real functions spaces. Formalized Mathematics, 1(3):555-561, 1990.

[15] Yasunari Shidama and Noboru Endou. Integral of real-valued measurable function. Formalized Mathematics, 14(4):143-152, 2006, doi:10.2478/v10037-006-0018-8.

[16] Yasunari Shidama, Hikofumi Suzuki, and Noboru Endou. Banach algebra of bounded functionals. Formalized Mathematics, 16(2):115-122, 2008, doi:10.2478/v10037-008-0017-

[17] Andrzej Trybulec. Binary operations applied to functions. Formalized Mathematics, 1(2):329-334, 1990.

[18] Zinaida Trybulec. Properties of subsets. Formalized Mathematics, 1(1):67-71, 1990.

[19] Edmund Woronowicz. Relations and their basic properties. Formalized Mathematics, 1(1):73-83, 1990.

[20] Edmund Woronowicz. Relations defined on sets. Formalized Mathematics, 1(1):181-186, 1990.

[21] Hiroshi Yamazaki, Yasunari Shidama, and Yatsuka Nakamura. Bessel's inequality. Formalized Mathematics, 11(2):169-173, 2003.

[22] Bo Zhang, Hiroshi Yamazaki, and Yatsuka Nakamura. The relevance of measure and probability, and definition of completeness of probability. Formalized Mathematics, 14(4):225-229, 2006, doi:10.2478/v10037-006-0026-8.

Received March 16, 2010 